
\title{
25 Research Square \\ Liquid-liquid equilibria for aromatic extraction systems using artificial neural networks
}

Saad Ahmed

University of Baghdad

Ammar Al-Dujaili ( $\square$ ah.aldujaili@gmail.com )

UJ: The University of Jordan https://orcid.org/0000-0002-1759-8585

\section{Research Article}

Keywords: Liquid-liquid equilibria, Neural network, Benzene, Pentane, Sulfolane, heptane

Posted Date: February 7th, 2022

DOI: https://doi.org/10.21203/rs.3.rs-1273720/v1

License: (c) (i) This work is licensed under a Creative Commons Attribution 4.0 International License. Read Full License 


\section{Abstract}

Phase equilibrium data regression is necessary to obtain the suitable values for modelling liquid-liquid extraction. It is important in equipment design, simulation, and optimization. Different factors may affect the accuracy of results. The present paper treats the nonlinear behavior of multicomponent liquid-liquid extraction using artificial neural networks. Two systems were studied, pentane-benzene- sulfolane system and heptane-benzene-tetraethylene glycol (TEG)-water system. Learning the first system in the neural networks doesn't gave good results while for the second equilibrium system, interaction terms were input, good values of mean square error and regression coefficient $\left(R^{2}\right)$ values achieved.

\section{Introduction}

The monocyclic aromatic hydrocarbons like benzene, toluene, xylenes are valuable chemicals in petrochemical industry. They are produced by catalytic reforming of C6-C9 Naphtha's, followed by solvent extraction with selective solvents like Sulfolane or tetra ethylene glycol mixed with other solvents [1]. Sometimes the extraction process is important to reduce components like benzene for environmental requirements [2-5].

Phase equilibria and the interaction between phases are important issues in separation processes for both scientists and engineers. With multicomponent systems calculation is tedious, this is quite reason to use neural networks for evaluation of such systems. Sharifi and Abbasi et al., applied neural networks (NN) for perturbed-chain statistical associating fluid theory equation of state to estimate the crossinteraction parameters for each binary pair in multicomponent system, whereas phase equilibrium calculations require huge computation costs [6].

To understand the distribution of such components in an extraction process, their liquid-liquid equilibrium (LLE) behavior between the aqueous and organic phase must be known [1]. Experimental data are usually correlated in. There was extensive work on modelling and simulation methods on benzene recovery like universal quasichemical (UNIQUAQ), universal quasichemical functional activity coefficients (UNIFAC) and non-random two-liquid (NTRL) [7]. Modelling of phase equilibria is essential for simulation and optimization of separation processes in the chemical industry. The accurate phase equilibrium data regressions depend on different factors such as the experimental quality, the selected model used to describe the excess Gibbs energy, and the correlation algorithm [8, 9].

\section{Theory}

\section{Artificial neural networks}

The human brain processes a large amount of data in a parallel manner through neurons approximately in one millisecond after firing and multitasks are performed ${ }^{5}$. The brain neuron structure and the artificial neuron structures are shown in Fig. 1. The artificial neuron is a trial to mimic the behavior of biological 
neurons [10]. Artificial neural network (ANN) and Biological neural network (BNN) can be interpreted in the similar methods [11].

In ANN, each input is multiplied by a factor called weight, the inputs multiplied by weights are summed and feed in a linear combination which makes the feed of hidden layer. The hidden layer neuron activates the input by an activation function (Fig. 2). Many types of activation functions existed [12], training $p$ neuron sometimes called perceptron and updating the weights in many numbers of trials. The network consists of a number of neurons in the input layer, and hidden layers, the output layer gives the output of the neural network.

\section{Neural networks applications in chemistry}

There are some applications of artificial neural networks in chemistry field as well as in chemical engineering [13]; prediction of chemical patterns [9], prediction of organic chemistry reactions [9], proton design $[14,15]$ and phase equilibrium of binary system [16].

\section{Building the neural network}

Two systems were studied in this paper, the first one pentane-benzene-sulfolane, which was experimentally studied by Cassel et al., [17] (Table 1). The second system was heptane-p-xylene-TEGwater studied by Wang et al., [14] (Table 2). A neural network of 10 neurons in the hidden layer used for learning the Cassel et. al., [17] shown in Fig. 3 and the neural network trained by experimental work of Wang et. al., [14] is shown in Fig. 4.

\section{Results And Discussion}

\section{Pentane-benzene-sulfolane}

Training the neural network for the mixture of pentane-benzene-sulfolane gave a good result from the first attempts, on the program default settings. Better results achieved when increasing the training sets as shown in Table 3. An evident decrease in the mean square error achieved by increase the training set, and the correlation factor $\mathrm{R}^{2}$ enhanced.

All data are tested in the neural network and the executed results were plotted for the pentane-benzenesulfolane mixture as shown in Table 4 and Fig. 5 . Figure 5 shows a zigzag shape line; the inflection could be attributed to the temperature factor. 
Table 1

Experimental liquid-liquid equilibrium composition for pentane-benzene-sulfolane system [16]

\begin{tabular}{|c|c|c|c|c|c|c|}
\hline \multirow[t]{2}{*}{$\mathrm{T}\left({ }^{\circ} \mathrm{C}\right)$} & \multicolumn{3}{|l|}{ Raffinate } & \multicolumn{3}{|l|}{ Extract } \\
\hline & Pentane & Benzene & Sulfolane & Pentane & Benzene & Sulfolane \\
\hline 17 & 0.88 & 0.00 & 99.12 & 99.34 & 0.00 & 0.66 \\
\hline 17 & 0.95 & 4.75 & 94.29 & 89.70 & 9.53 & 0.77 \\
\hline 17 & 1.09 & 9.32 & 89.60 & 79.81 & 19.01 & 1.18 \\
\hline 17 & 1.30 & 14.75 & 83.95 & 70.14 & 28.07 & 1.79 \\
\hline 17 & 1.50 & 21.34 & 77.16 & 60.80 & 36.40 & 2.80 \\
\hline 17 & 1.50 & 23.19 & 75.31 & 57.83 & 38.42 & 3.75 \\
\hline 17 & 1.67 & 28.21 & 70.13 & 49.54 & 45.71 & 4.75 \\
\hline 17 & 2.66 & 31.35 & 66.00 & 43.35 & 50.37 & 6.28 \\
\hline 17 & 4.33 & 39.29 & 56.39 & 33.24 & 58.31 & 8.45 \\
\hline 17 & 10.37 & 47.16 & 42.48 & 26.34 & 58.79 & 14.87 \\
\hline 25 & 0.88 & 0.00 & 99.12 & 99.29 & 0.00 & 0.71 \\
\hline 25 & 1.10 & 4.79 & 94.11 & 89.78 & 9.29 & 0.93 \\
\hline 25 & 1.12 & 9.72 & 89.16 & 80.50 & 18.36 & 1.14 \\
\hline 25 & 1.78 & 15.83 & 82.39 & 67.92 & 29.98 & 2.10 \\
\hline 25 & 2.06 & 21.48 & 76.46 & 61.03 & 36.50 & 2.47 \\
\hline 25 & 2.13 & 23.04 & 74.83 & 57.90 & 38.60 & 3.50 \\
\hline 25 & 2.50 & 29.01 & 68.49 & 47.22 & 47.50 & 5.28 \\
\hline 25 & 2.91 & 32.40 & 64.69 & 40.90 & 52.69 & 6.41 \\
\hline 25 & 4.18 & 39.50 & 56.32 & 31.67 & 57.12 & 11.21 \\
\hline 50 & 1.12 & 0.00 & 98.88 & 99.29 & 0.00 & 0.71 \\
\hline 50 & 1.28 & 5.05 & 93.67 & 88.64 & 10.50 & 0.86 \\
\hline 50 & 1.22 & 9.45 & 89.33 & 79.24 & 19.32 & 1.44 \\
\hline 50 & 1.55 & 16.04 & 82.41 & 67.67 & 28.87 & 3.46 \\
\hline 50 & 2.26 & 20.10 & 77.64 & 60.74 & 35.46 & 3.80 \\
\hline 50 & 2.65 & 23.26 & 74.09 & 55.79 & 38.67 & 5.54 \\
\hline
\end{tabular}




\begin{tabular}{|lllllll|}
\hline $\mathrm{T}\left({ }^{\circ} \mathrm{C}\right)$ & Raffinate & \multicolumn{5}{c|}{ Extract } \\
\cline { 2 - 7 } & Pentane & Benzene & Sulfolane & Pentane & Benzene & Sulfolane \\
\hline 50 & 2.65 & 30.37 & 66.98 & 46.04 & 47.92 & 6.04 \\
\hline 50 & 3.21 & 34.04 & 62.75 & 38.82 & 52.74 & 8.44 \\
\hline 50 & 4.08 & 39.70 & 56.22 & 29.88 & 56.02 & 14.10 \\
\hline
\end{tabular}

Table 2

Experimental liquid-liquid equilibrium composition for heptane-p-xylne-TEG-water system [17]

\begin{tabular}{|cllllllll|}
\hline No. & \multicolumn{7}{l}{ Raffinate phase } & \multicolumn{5}{c|}{ Extract phase } \\
\cline { 2 - 8 } & Heptane & $p$-Xylne & TEG & Water & Heptane & $p$-Xylne & TEG & Water \\
\hline 1 & 0.7325 & 0.2614 & 0.0037 & 0.0024 & 0.0119 & 0.0347 & 0.5817 & 0.3716 \\
\hline 2 & 0.4565 & 0.5268 & 0.0102 & 0.0065 & 0.00720 & 0.0531 & 0.5734 & 0.3663 \\
\hline 3 & 0.4593 & 0.5284 & 0.0075 & 0.0048 & 0.0103 & 0.0741 & 0.5587 & 0.3569 \\
\hline 4 & 0.6248 & 0.3692 & 0.0037 & 0.0024 & 0.00710 & 0.0473 & 0.557 & 0.3685 \\
\hline 5 & 0.5492 & 0.4204 & 0.0185 & 0.0018 & 0.0102 & 0.0595 & 0.5677 & 0.3625 \\
\hline 6 & 0.1617 & 0.8177 & 0.0125 & 0.008 & 0.00770 & 0.1255 & 0.5289 & 0.3379 \\
\hline 7 & 0.4299 & 0.5504 & 0.016 & 0.0037 & 0.0172 & 0.1215 & 0.7014 & 0.1589 \\
\hline 8 & 0.6172 & 0.3729 & 0.0077 & 0.0018 & 0.0227 & 0.0852 & 0.7265 & 0.1657 \\
\hline 9 & 0.3121 & 0.678 & 0.0081 & 0.0018 & 0.0291 & 0.1795 & 0.6444 & 0.147 \\
\hline 10 & 0.5442 & 0.412 & 0.0357 & 0.0064 & 0.0167 & 0.0877 & 0.7293 & 0.1663 \\
\hline 11 & 0.5408 & 0.4259 & 0.0271 & 0.0055 & 0.0205 & 0.0939 & 0.7212 & 0.1645 \\
\hline 12 & 0.4743 & 0.4911 & 0.0282 & 0.0077 & 0.0199 & 0.1071 & 0.7108 & 0.1525 \\
\hline 13 & 0.4627 & 0.4955 & 0.034 & 0.0067 & 0.0199 & 0.0958 & 0.7201 & 0.1642 \\
\hline
\end{tabular}

\section{Heptane-p-xylene-TEG-water system}

Training the neural network for the mixture of heptane- $p$-xylne-TEG-water system gave unsatisfied of mean square error and $\mathrm{R}^{2}$ values even with increasing hidden layer neurons. The problem is solved by adding the interaction between the organic components and a quite reasonable result achieved. Further 
enhancement achieved by increasing the input sets to the network. These trials are shown in Table 5. An evident decrease in the mean square error achieved by increase the training set, and the correlation factor $\mathrm{R}^{2}$ enhanced.

Table 3

Values of MSE and $\mathrm{R}^{2}$ for Training, Validation, and Testing

\begin{tabular}{|lllllll|}
\hline Results & Samples & MSE & $\mathbf{R}^{2}$ & Samples & MSE & $\mathbf{R}^{2}$ \\
\hline Training & 20 & 0.0259 & 0.9988 & 26 & 0.0852 & 0.9995 \\
\hline Validation & 4 & 0.0119 & 0.9962 & 1 & 0.0291 & 0.9999 \\
\hline Testing & 4 & 0.0199 & 0.9984 & 1 & 0.0632 & 0.9999 \\
\hline
\end{tabular}

Adding term for the interaction between heptane and $p$-xylene and M2N refers to adding another terms for the interaction between heptane and TEG and the interaction between $p$-xylene and TEG. Values of $\mathrm{R}^{2}$ enhanced from 0.9884 to 0.9978 . This enhancement is important for good fitting. Experimental and neural network modeling results are plotted in Fig. 6. 
Table 4

Experimental and model results for pentane-benzene-sulfolane system

\begin{tabular}{|c|c|c|c|c|c|c|}
\hline \multirow[t]{2}{*}{$\mathrm{T}\left({ }^{\circ} \mathrm{C}\right)$} & \multicolumn{3}{|l|}{ ANN } & \multicolumn{3}{|c|}{ Experimental } \\
\hline & Pentane\% & Benzene\% & Sulfolane\% & Pentane\% & Benzene\% & Sulfolane\% \\
\hline \multirow[t]{10}{*}{17} & 99.27155 & -0.08375 & 0.860660 & 0.00 & 0.00 & 0.66 \\
\hline & 89.76376 & 9.54814 & 0.726235 & 89.70 & 9.53 & 0.77 \\
\hline & 79.78906 & 19.40284 & 0.852626 & 79.81 & 19.01 & 1.18 \\
\hline & 69.94935 & 28.12000 & 1.897061 & 70.14 & 28.07 & 1.79 \\
\hline & 60.69366 & 36.21941 & 2.997793 & 60.80 & 36.40 & 2.80 \\
\hline & 57.99514 & 38.50091 & 3.409181 & 57.83 & 38.42 & 3.75 \\
\hline & 49.54848 & 45.58060 & 4.803447 & 49.54 & 45.71 & 4.75 \\
\hline & 43.69978 & 50.12378 & 6.135552 & 43.35 & 50.37 & 6.28 \\
\hline & 33.60577 & 57.84664 & 8.637892 & 33.24 & 58.31 & 8.45 \\
\hline & 26.38503 & 58.72607 & 14.82544 & 26.34 & 58.79 & 14.87 \\
\hline \multirow[t]{9}{*}{25} & 99.46963 & 0.18853 & 0.511169 & 99.29 & 0.00 & 0.71 \\
\hline & 89.44026 & 9.46105 & 1.224706 & 89.78 & 9.29 & 0.93 \\
\hline & 80.78937 & 18.26884 & 1.046113 & 80.50 & 18.36 & 1.14 \\
\hline & 68.21736 & 29.65856 & 2.193729 & 67.92 & 29.98 & 2.10 \\
\hline & 60.75473 & 36.87243 & 2.42173 & 61.03 & 36.50 & 2.47 \\
\hline & 58.48516 & 38.75858 & 2.787028 & 57.90 & 38.60 & 3.50 \\
\hline & 47.29987 & 47.24741 & 5.411572 & 47.22 & 47.50 & 5.28 \\
\hline & 40.68110 & 51.79155 & 7.429843 & 40.90 & 52.69 & 6.41 \\
\hline & 31.45940 & 57.07565 & 11.24073 & 31.67 & 57.12 & 11.21 \\
\hline \multirow[t]{7}{*}{50} & 99.30776 & 0.106843 & 0.589593 & 99.29 & 0.00 & 0.71 \\
\hline & 88.48189 & 10.42493 & 1.073134 & 88.64 & 10.50 & 0.86 \\
\hline & 79.46656 & 18.99350 & 1.538858 & 79.24 & 19.32 & 1.44 \\
\hline & 67.69917 & 29.01656 & 3.302533 & 67.67 & 28.87 & 3.46 \\
\hline & 60.91898 & 34.69250 & 4.377269 & 60.74 & 35.46 & 3.80 \\
\hline & 56.13480 & 38.87121 & 4.990123 & 55.79 & 38.67 & 5.54 \\
\hline & 45.96211 & 48.22792 & 6.076392 & 46.04 & 47.92 & 6.04 \\
\hline
\end{tabular}




\begin{tabular}{llllll|}
38.38134 & 52.63656 & 9.106049 & 38.82 & 52.74 & 8.44 \\
\hline 29.01228 & 57.07592 & 13.80303 & 29.88 & 56.02 & 14.10 \\
\hline
\end{tabular}

Table 5 The various network selected to enhance the values of MSE and $\mathrm{R}^{2}$ for heptane-p-xylene-TEGwater system

\begin{tabular}{|llllllll|}
\hline & & M1 & & M1N & \multicolumn{3}{l|}{ M2N } \\
\hline & No. & MSE & $\mathrm{R}^{2}$ & MSE & $\mathrm{R}^{3}$ & MSE & $\mathrm{R}^{4}$ \\
\hline Training & 9 & 0.00014 & 0.9884 & 0.00003 & 0.9974 & 0.00009 & 0.9993 \\
Validation & 2 & 0.01063 & 0.9518 & 0.00956 & 0.9133 & 0.00063 & 0.9964 \\
\hline Testing & 2 & 0.00555 & 0.9334 & 0.00654 & 0.9468 & 0.00457 & 0.9664 \\
\hline Training & 11 & 0.00124 & 0.9901 & 0.00003 & 0.9998 & 0.00005 & 0.9996 \\
\hline Validation & 1 & 0.00438 & 0.9815 & 0.00013 & 0.9997 & 0.00008 & 0.9998 \\
\hline Testing & 1 & 0.00492 & 0.9843 & 0.00026 & 0.9986 & 0.00041 & 0.9991 \\
\hline
\end{tabular}

\section{Conclusions}

It is concluded that feed forward neural networks model is suitable for modeling experimental data of liquid-liquid equilibrium. A system pentane-benzene sulfolane was well simulated in the multilayer perceptron neural network. Neural network with Levenberg-Marquardt Algorithm to fit experimental data with 10 neurons in the input layer and 4 hidden layer neurons was efficient for the training. For the heptane-p-xylene-TEG-water system, the interaction between organic components of the mixture affects the simulation results by neural networks.

\section{Declarations}

\section{Competing interests:}

The authors declare no competing interests.

\section{References}

1. Busca G (2021) Energies 14(13):4061-4075

2. Janitz AE, Campbell JE, Magzamen S, Pate A, Stoner JA, Peck JD (2017) Environ Res 158:167-173

3. Houot J, Marquant F, Goujon S, Faure L, Honoré C, Roth MH, Denis Hémon D, Clavel J (2014) Am J Epidemiology 182(8):685-693 
4. Linet MS, Yin SN, Gilbert ES, Dores GM, Hayes RB, Vermeulen R, Tian HY, Lan Q, Portengen L, Ji BT, Li GL, Rothman N (2015) Int J Cancer 137(9):2184-2193

5. Stenehjem JS, Kjærheim K, Bråtveit M, Samuelsen SO, Adesi FB, Rothman N, Lan Q, Grimsrud TK (2015) British J Cancer 112(9):1603-1612

6. Wang K, Luo J, Wei Y, Wu K, Li J, Chen Z (2020) J Comput Phys 401(15):1-22

7. Usman MA, Fagoroye OK, Ajayi TO, Kehinde AJ (2020) Appl Petrochem Res 10(3):125-137

8. Yu CH, Buehler MJ (2020) APL Bioeng 4(1):016108

9. Cova TFGG, Pais AACC (2019) Frontiers in Chem 7:1-44

10. Parente E (2011) Analytical Methods: Multivariate Statistical Tools for Chemometrics. in Encyclopedia of Dairy Sciences. Second Edition

11. Xu Y, Vaziri-Pashkam M (2021) Nat Commun 12(1):2065

12. Sharma S, Sharma S, Athaiya A (2020) Int J Eng Appl Sci Technol 4(12):310-316

13. Sharifi S (2121) J Chem Reviews 3(4):273-289

14. Wang J, Cao H, Zhang JZH, Qi Y (2018) Sci Reports 8(1):8

15. Oreški S, Zupan J, Glavič P (2002) Chem Biochem Eng Quarterly 16(2):41-57

16. Song Y, Zhou H, Wang P, Yang M (2019) J Chem Thermodyn 135:86-96

17. Cassell GW, Dural N, Hines AL (1989) Indus Eng Chem Res 28(9):1369-1374

\section{Figures}

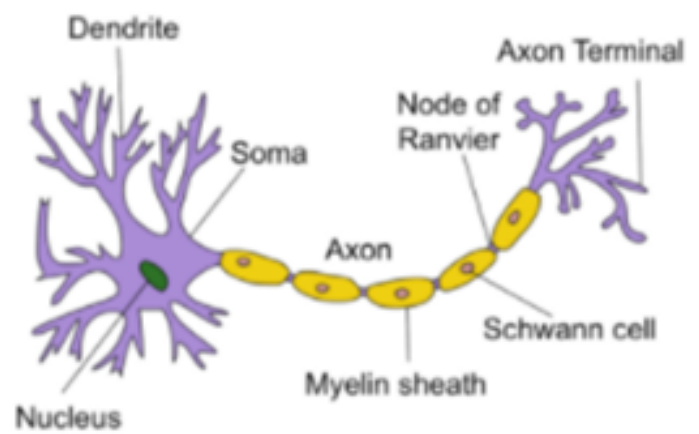

Structure of a typical neuron

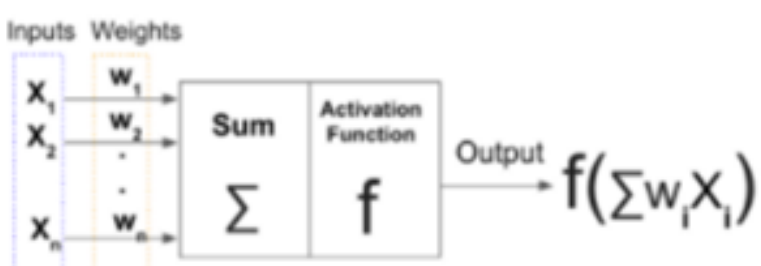

Structure of artificial neuron

\section{Figure 1}

The brain neuron structure and the artificial neuron structures 


\begin{tabular}{|c|c|c|c|}
\hline Name & Plot & Equation & Derivative \\
\hline Identity & & $f(x)=x$ & $f^{\prime}(x)=1$ \\
\hline Binary step & & $f(x)=\left\{\begin{array}{lll}0 & \text { for } & x<0 \\
1 & \text { for } & x \geq 0\end{array}\right.$ & $f^{\prime}(x)=\left\{\begin{array}{lll}0 & \text { for } & x \neq 0 \\
? & \text { for } & x=0\end{array}\right.$ \\
\hline $\begin{array}{l}\text { Logistic (a.k. a } \\
\text { Soft step) }\end{array}$ & & $f(x)=\frac{1}{1+e^{-x}}$ & $f^{\prime}(x)=f(x)(1-f(x))$ \\
\hline TanH & & $f(x)=\tanh (x)=\frac{2}{1+e^{-2 x}}-1$ & $f^{\prime}(x)=1-f(x)^{2}$ \\
\hline ArcTan & & $f(x)=\tan ^{-1}(x)$ & $f^{\prime}(x)=\frac{1}{x^{2}+1}$ \\
\hline $\begin{array}{l}\text { Rectified } \\
\text { Linear Unit } \\
\text { (ReLU) }\end{array}$ & & $f(x)=\left\{\begin{array}{lll}0 & \text { for } & x<0 \\
x & \text { for } & x \geq 0\end{array}\right.$ & 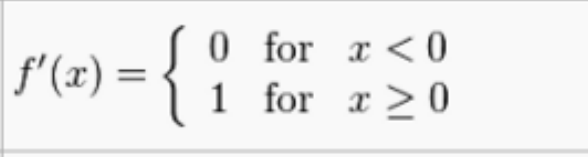 \\
\hline $\begin{array}{l}\text { Paraneteric } \\
\text { Rectified } \\
\text { Linear Unit } \\
\text { (PReLU) }^{[2]}\end{array}$ & & $f(x)=\left\{\begin{aligned} \alpha x & \text { for } \quad x<0 \\
x & \text { for } \quad x \geq 0\end{aligned}\right.$ & $f^{\prime}(x)= \begin{cases}\alpha & \text { for } \quad x<0 \\
1 & \text { for } \quad x \geq 0\end{cases}$ \\
\hline $\begin{array}{l}\text { Exponential } \\
\text { Linear Unit } \\
\text { (ELU) }{ }^{[3]}\end{array}$ & & $f(x)=\left\{\begin{array}{rll}\alpha\left(e^{x}-1\right) & \text { for } & x<0 \\
x & \text { for } & x \geq 0\end{array}\right.$ & $f^{\prime}(x)=\left\{\begin{array}{rll}f(x)+\alpha & \text { for } & x<0 \\
1 & \text { for } & x \geq 0\end{array}\right.$ \\
\hline SoftPlus & & $f(x)=\log _{e}\left(1+e^{x}\right)$ & $f^{\prime}(x)=\frac{1}{1+e^{-x}}$ \\
\hline
\end{tabular}

\section{Figure 2}

The famous activation functions usually used 


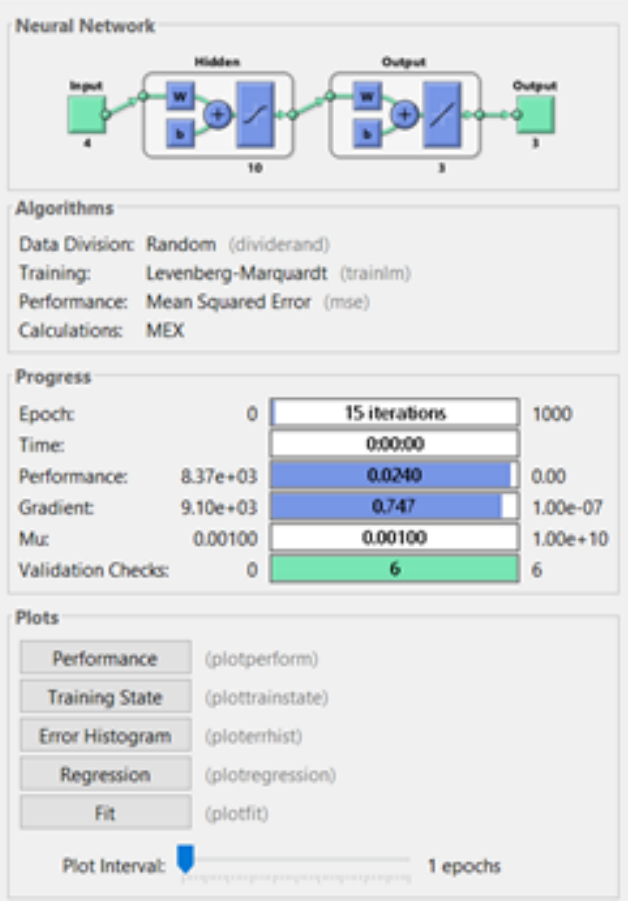

Figure 3

The neural network constructed for training mixture of pentane-benzene- sulfolane system

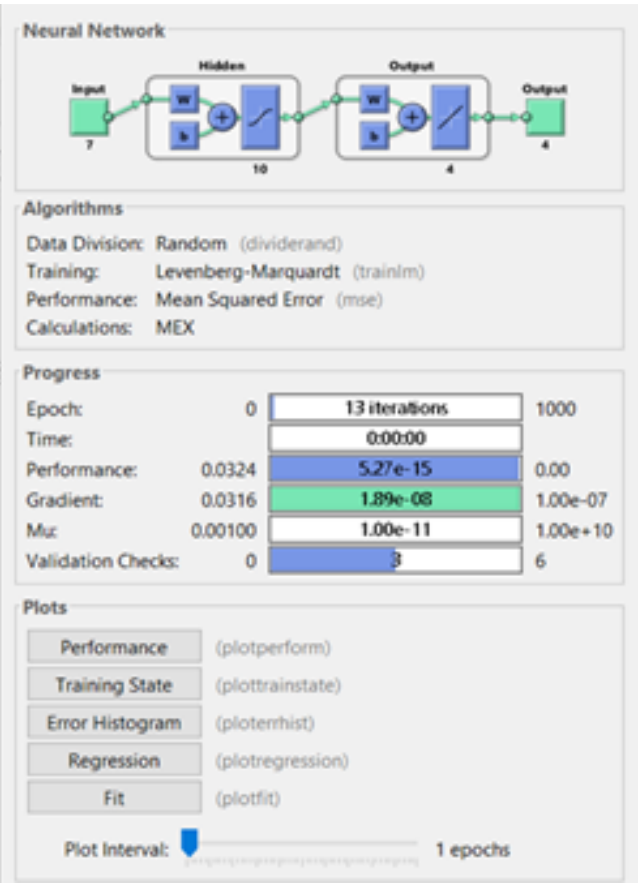

Figure 4

The neural network constructed for training mixture of heptane- $p$-xylene-TEG-water system 

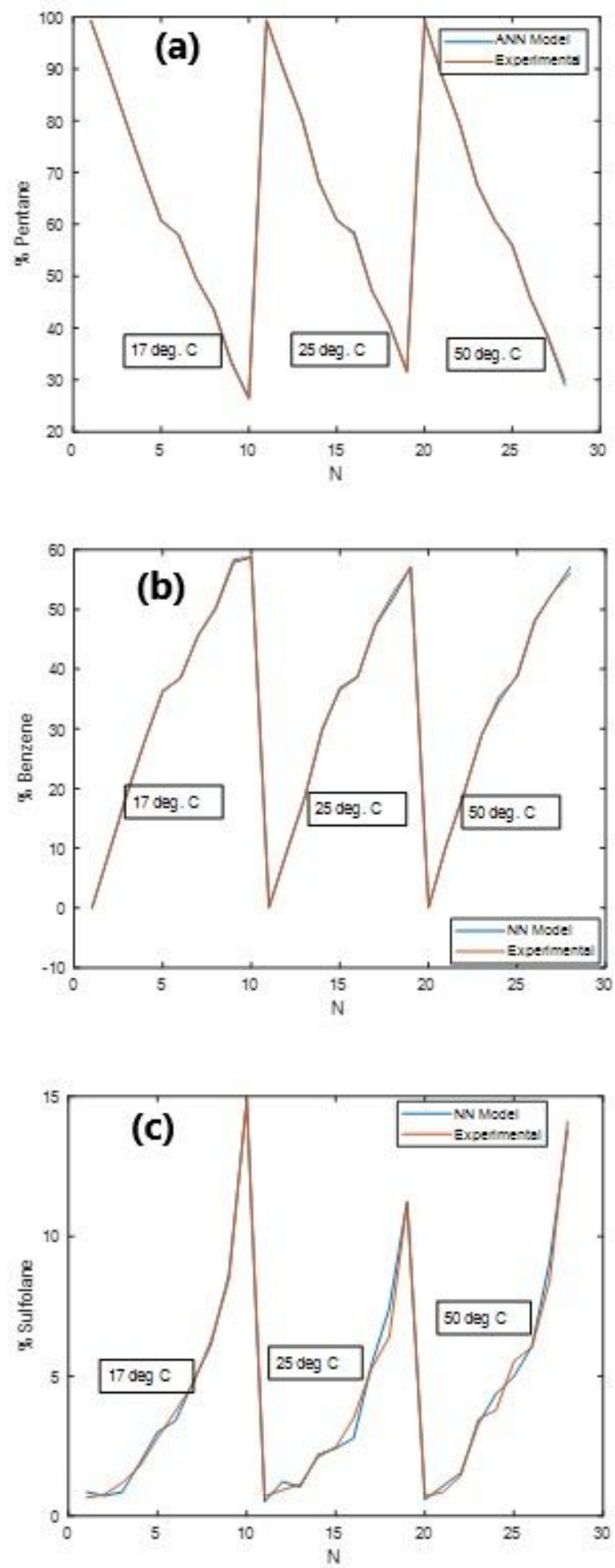

Figure 5

The ANN model and experimental values of (a) pentane, (b) benzene and (c) sulfolane 

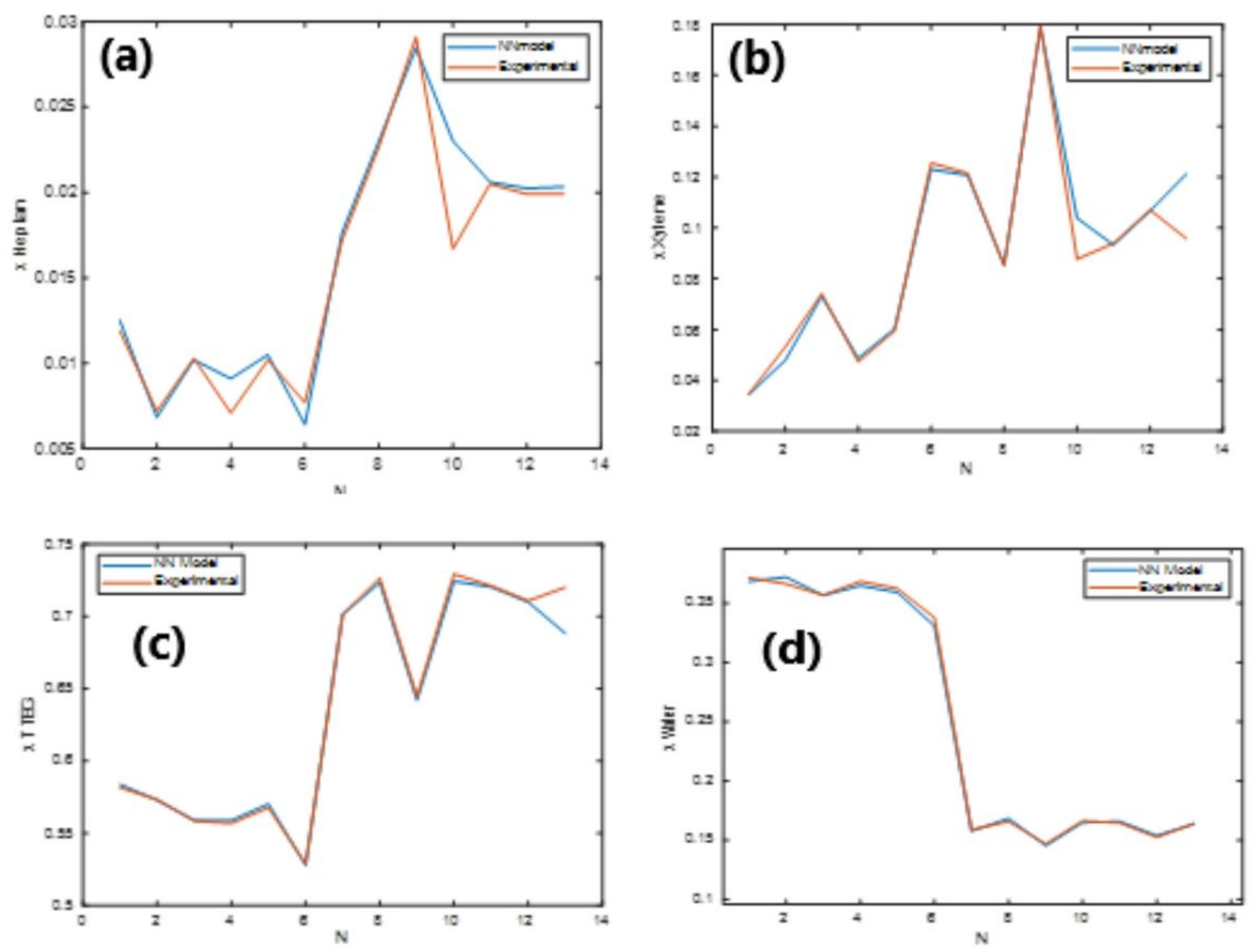

Figure 6

The ANN model and experimental values of (a) heptane, (b) p-xylene, (c) TEG and (d) water 